\title{
Emodin and rhein in Cassia tora ameliorates activity of mitochondrial enzymes involved in oxidative phosphorylation in the retina of diabetic mice
}

\author{
Eun $\mathrm{Ko}^{1 \dagger}$, Min Young Um ${ }^{2 \dagger}$, Taewon $\mathrm{Han}^{2}$, Sooim Shin ${ }^{1,3^{*}}$ and Moonsung Choi ${ }^{4,5^{*}}$
}

\begin{abstract}
Cassia tora is an annual herb, which has pharmacological effects such as antioxidant, hypolipidemic, and antidiabetic effects. Accordingly, its effect on diabetes has been well-studied. However, it is unclear whether it has an effect on mitochondrial dysfunction associated with diabetes. In this study, the effects of emodin and rhein in C. tora seed ethanolic extract (ER/CSEE) on retinal mitochondrial function were examined in high-fat diet (HFD)-fed mice. HFDfed mice exhibited decreased mitochondrial function followed by compensatory increase in the expression levels of mitochondrial enzymes. However, ER/CSEE treatment for 12 weeks ameliorated the activity of retinal mitochondrial complexes and reduced the expression level of enzymes involved in oxidative phosphorylation, except that of complex II and citrate synthase in citric acid cycle. This suggests that repairing capacity of enzymes in electron transport chain and citric acid cycle of mitochondria are different in response to the metabolic state. Therefore, it concluded that emodin and rhein play a pharmacological role in fat metabolism by influencing activities of enzymes in citric acid cycle linked with beta-oxidation in retina.
\end{abstract}

Keywords: Cassia tora, Emodin, Rhein, Mitochondria, Oxidative phosphorylation, Type 2 diabetes

\section{Introduction}

Type 2 diabetes (T2D) is the most threatening metabolic disease, affecting more than $10 \%$ population worldwide [1]. A typical symptom of T2D is abnormal glycemic regulation, and the long-term progression of T2D results in severe complications in critical organs in the human body [2]. Mitochondrion is the central organelle for glucose catabolism, in which adenosine triphosphate molecules

\footnotetext{
*Correspondence: sooim.shin@jnu.ac.kr; mschoi@seoultech.ac.kr ${ }^{\dagger}$ Eun Ko and Min Yong Um contributed equally to this work ${ }^{1}$ Department of Biotechnology and Bioengineering, College of Engineering, Chonnam National University, 77 Yongbong-ro, Buk-gu, Gwangju 61186, Republic of Korea

${ }^{4}$ Department of Optometry, College of Energy and Biotechnology, Seoul National University of Science and Technology, Seoul 01811, Republic of Korea

Full list of author information is available at the end of the article
}

are produced from glucose-delivered metabolites in the process of oxidative phosphorylation (OXPHOS) [3]. Increased concentration of glucose in blood results in increased glucose uptake by the organs, thus leading to an overload of metabolites in the mitochondria. This can further lead to an imbalance in metabolism and elevate oxidative stress [4]. Several organs malfunction due to diabetes; particularly, metabolic imbalance and unregulated angiogenesis in the eye can cause diabetic retinopathy [5]. Diabetic retinopathy is the most frequent cause of blindness, with its prevalence reported in $60 \%$ of patients with T2D [6]. The pathogenesis of retinopathy involves swelling of the macula and proliferation of retinal blood vessels that eventually disrupt clear vision $[7,8]$.

Cassia tora is well known in East Asia as an alternative medicine to treat diabetes and retinal dysfunction. Other than this, the whole plant, leaves, roots, and seeds 
of $C$. tora have been used as a traditional medicine to treat various diseases such as psoriasis, skin diseases, and cardiovascular diseases [9]. The extract of C. tora contains pharmacologically useful phenolic compounds such as anthraquinones, naphthopyrones, naphthalenes, and hydroanthracenes and their glucosides [10, 11]. In a previous study, when the butanol-extracted fraction of $C$. tora seeds was administrated to streptozotocin-induced diabetic rats, the diabetes was ameliorated by the reduction of postprandial blood glucose and insulin secretion [12]. In addition, $C$. tora seed extract was shown to have hypolipidemic activity [13]. However, the effect of $C$. tora seeds on mitochondrial dysfunction associated with T2D are poorly examined. Therefore, we assessed the efficacy of emodin and rhein in $C$. tora seeds ethanolic extract (ER/CSEE) on the retinal health of mice with diabetes induced by a high-fat diet (HFD). Specifically, we examined the effect of ER/CSEE treatment on the mitochondrial function of the retina by measuring the activity of enzymes involved in OXPHOS thereby identify the linkage between the $C$. tora and functional improvement of mitochondria.

\section{Materials and methods}

\section{Preparation of $C$. tora seed extract}

Cassia tora seeds were bought from a commercial supplier Gyeongdong market (Seoul, Republic of Korea). The seeds of $C$. tora were ground into a powder with particle size of 40 mesh, and their extract was prepared in $70 \%$ ethanol. The extract was filtered and concentrated by rotary evaporation (Heidoph, LABORATA 4000, Schwabach, Germany). The concentrate was freeze dried and stored at $-20^{\circ} \mathrm{C}$ till further analysis.

\section{High-performance liquid chromatography for the quantification of Emodin and Rhein}

CSEE was subjected to high performance liquid chromatography (HPLC; 1290 Infinity II, Agilent Technologies, USA) to quantify the active compounds. Dried CSEE was dissolved in ethanol and heated at $60{ }^{\circ} \mathrm{C}$ for $20 \mathrm{~min}$. This was followed by sonication, and further, the extract was filtered using a membrane filter of pore size $0.45 \mu \mathrm{m}$. A total of $10 \mu \mathrm{L}$ sample was loaded to $\mathrm{C}_{18}(4.60 \times 250 \mathrm{~mm})$ column in $\mathrm{CH}_{3} \mathrm{CN}: 10 \mathrm{mM} /$ phosphate buffer (50:50 ratio; $\mathrm{pH} 2.6$ ) with flow rate of $0.7 \mathrm{~mL} / \mathrm{min}$.

\section{Animals and diets}

All procedures were performed according to Guide for Institutional Animal Care and Use Committee of the Korea Food Research Institute (KFRI-IACUC, KFRIM-16037). In this study, 5-week-old male C57BL/6 J mice (Central Lab. Animal Inc., Seoul, Republic of Korea) were maintained in a cage at $21-25^{\circ} \mathrm{C}, 50-60 \%$ humidity, $12 \mathrm{~h}$ each of light and dark cycles, and ad libitum access to food and water. After acclimatization period of 1 week, the mice were divided into three groups depending on the diet type. They were as follows: normal diet (ND; 10\% calories corresponding to fats, Research diets Inc., New Brunswick, USA), HFD (45\% calories corresponding to fats, Research diets Inc., New Brunswick, USA), and HFD with $0.1 \%$ ER/CSEE (w/w). The diet was maintained for 12 weeks. After the experimental period, blood was obtained from the retro-orbital sinus of mice in the fasting state. The eyes were removed, weighed, immediately snap-frozen in liquid $\mathrm{N}_{2}$, and stored at $-80{ }^{\circ} \mathrm{C}$ till further analysis.

\section{Assessment of mitochondrial oxidative phosphorylation}

Using homogenized eyes, individual activity of complexes I, II, III, and IV was measured by spectrophotometry using S-3100 UV-Vis Spectrophotometer (Scinco, Seoul, Republic of Korea) [14]. The activity of complex I [nicotinamide adenine dinucleotide (NADH) dehydrogenase] was assessed using $\mathrm{NADH}$ and ubiquinone as substrates in $50 \mathrm{mM}$ potassium phosphate at $\mathrm{pH} 7.5$ containing $3 \mathrm{mg} / \mathrm{mL}$ bovine serum albumin (BSA), $0.1 \mathrm{mM}$ $\mathrm{NADH}, 0.3 \mathrm{mM} \mathrm{KCN}$, and $0.06 \mathrm{mM}$ ubiquinone. The reaction for the complex I was monitored by checking absorbance at $340 \mathrm{~nm}$; decrease in absorbance indicated oxidation of NADH. The activity of complex II (succinate dehydrogenase) was assessed using succinate and ubiquinone as substrates in $50 \mathrm{mM}$ potassium phosphate at pH 7.5 containing $1 \mathrm{mg} / \mathrm{mL}$ BSA, $0.05 \mathrm{mM}$ decylubiquinone, $20 \mathrm{mM}$ succinate, $0.3 \mathrm{mM} \mathrm{KCN}$, and $0.015 \%(\mathrm{w} / \mathrm{v})$ 2,6-dichlorophenolindolphenol (DCPIP). The reaction for the complex II was monitored by measuring absorbance at $600 \mathrm{~nm}$; decrease in absorbance indicated reduction of DCPIP. The activity of complex III (cytochrome bc1 complex) was assessed using ubiquinol and ferric form of cytochrome $\mathrm{c}$ as substrates in $50 \mathrm{mM}$ potassium phosphate at $\mathrm{pH} 7.5$ containing $2.5 \%(\mathrm{v} / \mathrm{v})$ Tween ${ }^{\circledR} 20$, $0.3 \mathrm{mM} \mathrm{KCN}, 0.1 \mathrm{mM}$ decylubiquinol, $0.1 \mathrm{mM}$ ethylenediaminetetraacetic acid, and $0.075 \mathrm{mM}$ cytochrome c. The reaction for the complex III was monitored by measuring absorbance at $550 \mathrm{~nm}$; increase in absorbance indicated reduction of cytochrome c. The activity of complex IV (cytochrome c oxidase) was assessed using $0.06 \mathrm{mM}$ ferrous form of cytochrome $\mathrm{c}$ as a substrate in $10 \mathrm{mM}$ potassium phosphate at $\mathrm{pH}$ 7.0. The reaction for the complex IV was monitored by measuring absorbance at $550 \mathrm{~nm}$; decrease in absorbance indicated oxidation of cytochrome c. Citrate synthase was quantified using acetyl $\mathrm{CoA}$ and oxaloacetate as substrates in $20 \mathrm{mM}$ Tris buffer at $\mathrm{pH} 8.0$ with $0.2 \%(\mathrm{v} / \mathrm{v})$ triton $\mathrm{X}-100,0.3 \mathrm{mM}$ acetyl CoA, $0.5 \mathrm{mM}$ oxaloacetate, and $0.1 \mathrm{mM} 5,5^{\prime}$-dithiobis-(2-nitrobenzoic acid) (DTNB). 
The reaction was monitored by measuring absorbance at $412 \mathrm{~nm}$; increase in absorbance indicated reduction of DTNB to 2-nitro-5-thiobenzoic acid (TNB). All chemicals used for the assays were purchased from Sigma Aldrich (St. Louis, USA).

\section{Western blotting}

Mitochondria was isolated from homogenized eyes using Mitochondria Isolation Kit for Tissue (Thermo Fisher Scientific, Waltham, USA). Isolated mitochondria was loaded on $12 \%$ gel prepared using $\mathrm{TGX}^{\mathrm{TM}}$ FastCast $^{\mathrm{TM}}$ Acrylamide Kit (Bio-Rad, Hercules, USA), and isolated proteins were transferred to polyvinylidene fluoride (PVDF) membrane (Bio-Rad) using Trans-Blot Turbo Transfer system (25 V and 1A for 30 min: Turbo-Standard). The membrane was blocked with $2.5 \%$ BlottingGrade Blocker (Non-fat dry milk, Bio-Rad) in TBST buffer $\left(20 \mathrm{mM}\right.$ Tris, $150 \mathrm{mM} \mathrm{NaCl}$, and $0.1 \%$ Tween ${ }^{\circledR} 20$; $\mathrm{pH} 7.5)$ for $1 \mathrm{~h}$ at $26^{\circ} \mathrm{C}$ followed by overnight incubation at $4{ }^{\circ} \mathrm{C}$ with two primary antibodies: anti-OxPhos (1:500 dilution; Anti-OxPhos complex kit; Invitrogen, Carlsbad, USA) and prohibitin (1:500 dilution; Santacruz, Dallas, USA). Further, the membrane was washed several times with TBST buffer and incubated with goat anti-mouse IgG $(\mathrm{H}+\mathrm{L})$ secondary antibody (1:3000 dilution) with horseradish peroxidase-conjugate (Invitrogen) for $1.5 \mathrm{~h}$ at $26{ }^{\circ} \mathrm{C}$. The protein complexes were visualized using Clarity ${ }^{\mathrm{TM}}$ Western ECL Substrate (Bio-rad) on ChemiDoc System from Bio-rad. The samples were analyzed using Image Lab 6.0. (Bio-rad).

\section{Statistical analysis}

Results are expressed as the means or as the mean \pm SD. One-way analysis of variance (ANOVA) with post hoc Tukey test was used for statistical analysis using GraphPad Prism 8.0.1 software (GraphPad, Inc., La Jolla, USA). A p value of $<0.01$ was considered statistically significant.

\section{Results}

Identification of emodin and rhein in CSEE

Emodin and rhein are the anthraquinone metabolites known to have pharmacological effects [15-18]. For the quantification of them in CSEE, HPLC was performed. Emodin and rhein were found in CSEE, accounting for a total concentration of $8.51 \%$ (data not shown).

\section{ER/CSEE alleviated a compensatory increase in mitochondrial enzymes induced by HFD}

To assess the changes in the expression level of mitochondrial enzymes, western blotting was performed. The tendency of expression level change depending on the diet group was similar for all the mitochondrial complexes. Compared with the normal diet group, the HFD group exhibited increased expression levels of mitochondrial complexes I-V. However, administration of ER/CSEE to HFD mice resulted in a decrease in the expression levels of mitochondrial complexes, bringing the levels similar to those of normal diet group (Fig. 1). In a previous study, beta-cell mass and expression level of a specific protein exhibited compensatory increase in response to early diabetes [19]. This compensatory increase is the result of cellular response to ameliorate functions that might be disrupted by HFD [20]. Therefore, it can be assumed that the administration of ER/CSEE successfully repaired HFD-induced distorted metabolism in retinal cells or blocked the process of disruption.

\section{ER/CSEE recovered the HFD-induced disrupted function of mitochondrial enzymes involved in OXPHOS}

The activity of mitochondrial enzymes involved in OXPHOS was investigated in the three mice groups. The activity of all mitochondrial enzymes reduced after HFD in comparison with normal diet. However, when ER/CSEE was administrated to HFD-fed mice, activity of some of the mitochondrial enzymes was drastically recovered (Fig. 2). In case of complexes I and IV, mitochondrial activity was recovered and brought to the level similar to that of normal diet group. For complex III, although its activity in HFD with ER/CSEE group exhibited a slight decrease compared with ND, it was recovered to a considerable extent in comparison with that in HFD group.

\section{HFD-induced disrupted function of enzymes in the citric acid cycle was not recovered by ER/CSEE}

Though the activity of mitochondrial complexes I, III, and IV was recovered by ER/CSEE after HFD, that of complex II and citrate synthase did not exhibit any specific change compared with that in HFD group (Fig. $2 b$ and e). Mitochondrial complex II and citrate synthase are the enzymes involved in the citric acid cycle. NADH and $\mathrm{FADH}_{2}$, which act as crucial coenzymes for mitochondrial complexes I and II, are produced in citric acid cycle. In normal glucose metabolism, glucose is converted into pyruvate through glycolysis. Before entering the citric acid cycle, acetyl CoA is produced by pyruvate dehydrogenase from pyruvate. In lipid metabolism, triglyceride breaks down into glycerol and fatty acids. Fatty acids are metabolized through beta-oxidation to produce NADH, $\mathrm{FADH}_{2}$, and acetyl CoA. Further, acetyl CoA enters the citric acid cycle. When diet contains high portion of fats, extra energy is stored in the form of triglycerides resulting in a high influx of fatty acids for beta-oxidation. It leads to increased production of acetyl $\mathrm{CoA}$ as a substrate of the citric acid cycle, resulting in overload of metabolites in the citric acid cycle. Consequently, it may 

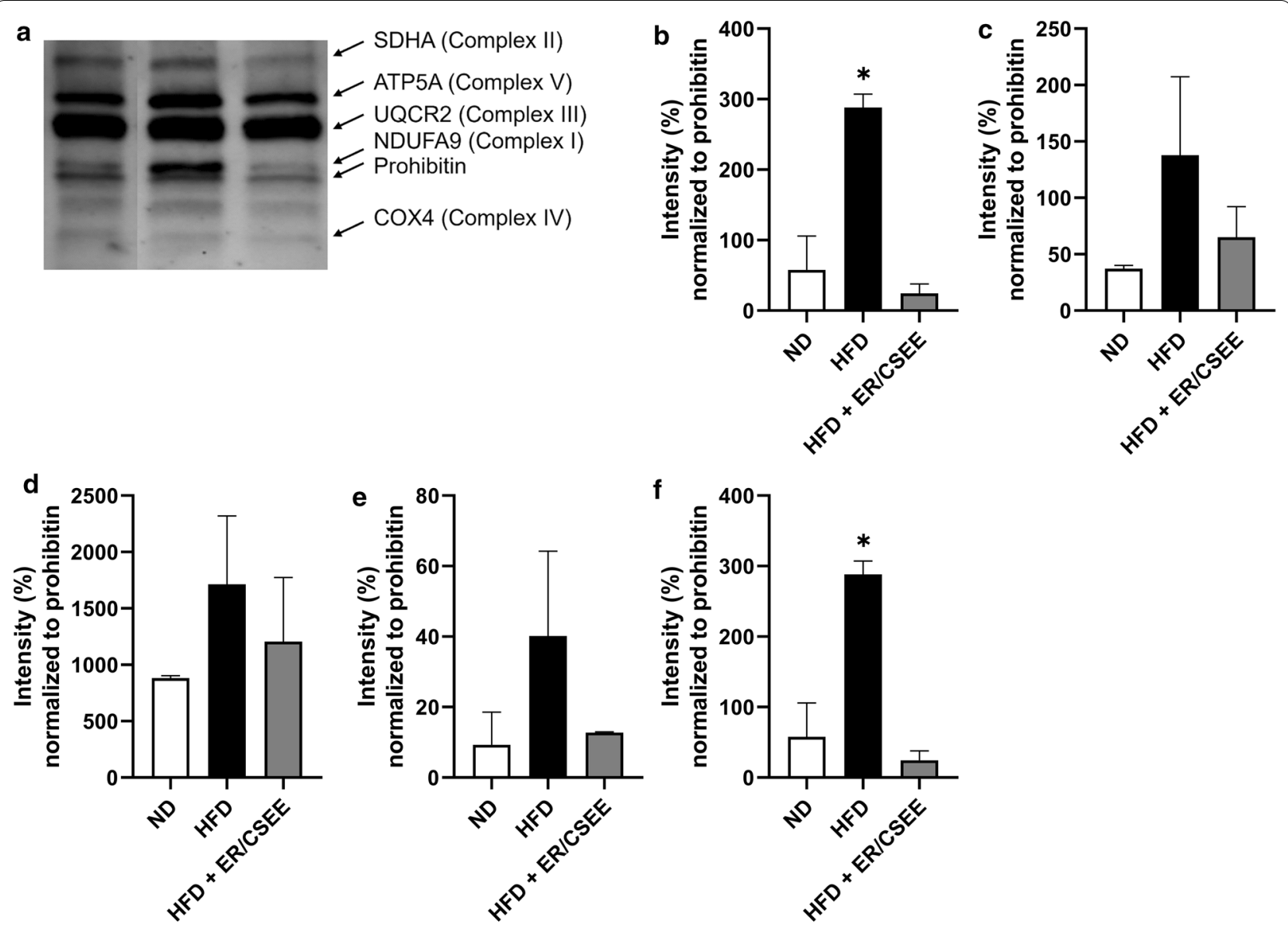

Fig. 1 Expression level of mitochondrial enzymes involved in oxidative phosphorylation (OXPHOS). a Levels of mitochondrial complexes visualized by western blotting. $\mathbf{b}$ Normalized expression level of mitochondrial complex I. c Normalized expression level of mitochondrial complex II. d Normalized expression level of mitochondrial complex III. e Normalized expression level of mitochondrial complex IV. $\mathbf{f}$ Normalized expression level of mitochondrial complex V. The values are given as mean \pm standard deviation ( $n=2$ for ND; $n=3$ for HFD; $n=2$ for HFD + ER/CSEE). Data among groups were compared using one-way analysis of variance $\left({ }^{*} P<0.01\right)$

cause disruption of functions of enzymes involved in the citric acid cycle.

\section{Discussion}

The seeds of $C$. tora, as alternative medicine, are known in East Asia for their effect on retinal health and diabetes [9]. To identify the function of emodin and rhein in C. tora on diabetic eyes, we investigated their effect on mitochondrial function in the eyes of mice with diabetes. Our previous research shows that blood index and expression level of mitochondrial enzymes, the inclusion of ER/CSEE with HFD diet prevented the deleterious effects of HFD diet. Moreover, mice fed with HFD and ER/CSEE exhibited similar levels of mitochondrial enzymes as those in mice fed with normal diet. Administration of ER/CSEE to HFD-fed mice exhibited decreased levels of fasting glucose and insulin, HOMA-IR, and increased adiponectin secretion compared with HFD-fed mice [21]. An earlier study reported that hyperinsulinemia caused by T2D resulted in a modified mitochondrial function causing increase in maximal OXPHOS capacity of oxygen consumption in diabetic Nile rats to compensate metabolic imbalance [22]. However, continuation of this modified mitochondrial function for 2 months resulted in compromising the integrity of the mitochondrial outer membrane. Finally, after 12 months, pericyte loss, the symptom of diabetic retinopathy, was observed. Additionally, other studies reported that diabetesinduced mitochondrial dysfunction was observed with oxidative stress and mutation in superoxide dismutase, and mitochondrial compensation in T2D preceded severe complications in the retina [23-25]. In this study, mitochondrial compensation was also observed in the form of increased expression level of enzymes involved in OXPHOS in HFD group. The process of OXPHOS is divided into the citric acid cycle and electron transport 

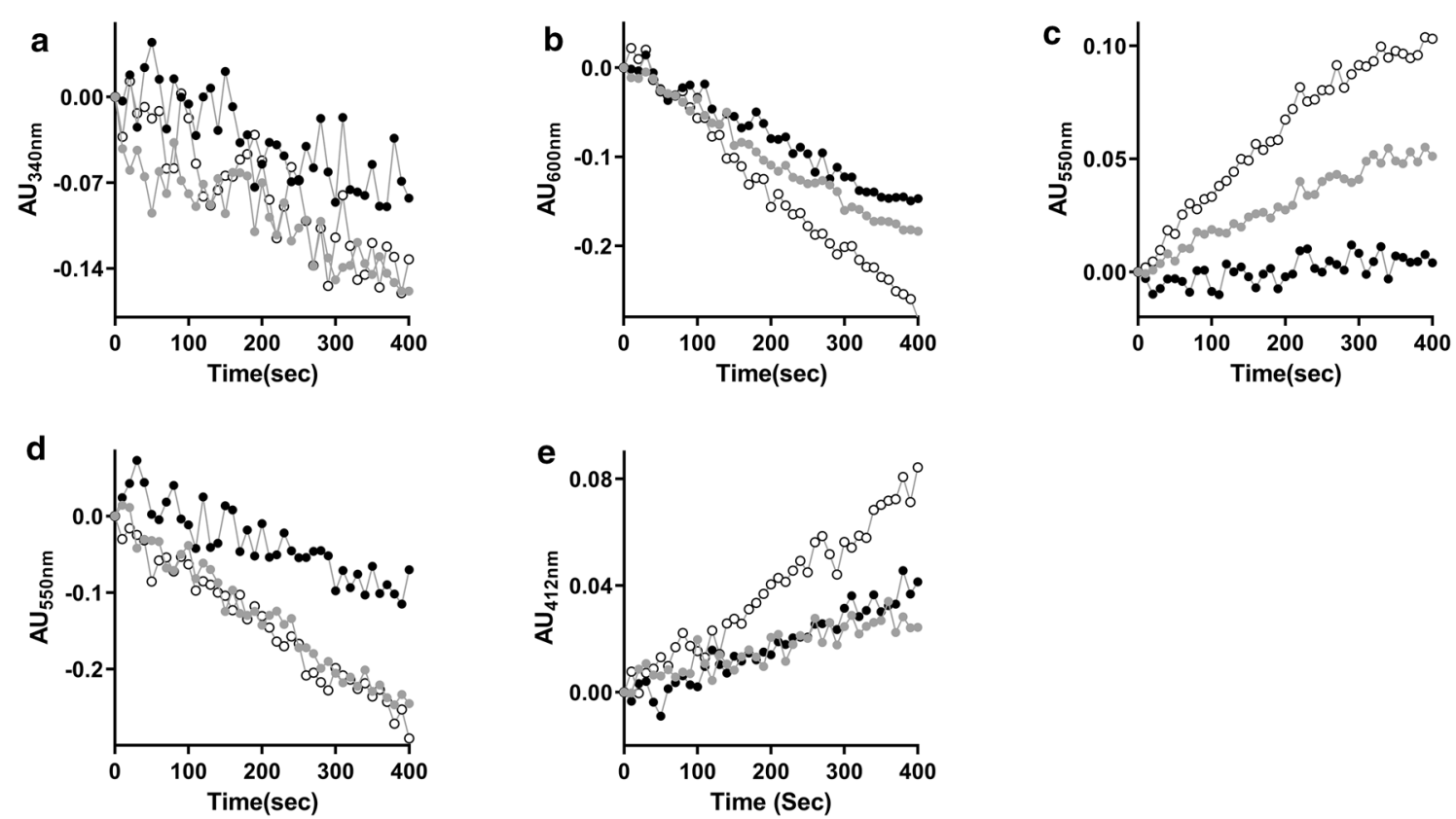

Fig. 2 Activity of mitochondrial complexes and citrate synthase involved in OXPHOS. a Activity of mitochondrial complex I. b Activity of mitochondrial complex II. c Activity of mitochondrial complex III. d Activity of mitochondrial complex IV. e Activity of mitochondrial citrate synthase. Activities in ND are shown as white dots, HFD as black dots, and HFD + ER/CSEE as gray dots. Values are given as means ( $n=7$ for all groups)

chain. In HFD with ER/CSEE group, the expression levels of mitochondrial enzymes of the electron transport chain were similar to those in normal diet group (Fig. 1). This indicated that ER/CSEE successfully prevented the metabolic imbalance in the early stage of diabetes, also averting mitochondrial compensation and diabetic retinopathy. In contrast, the activity of the enzymes involved in OXPHOS in mitochondria exhibited different patterns according to the category of the enzymes (Fig. 2). In HFD with ER/CSEE group, enzyme activity of the complexes I, III and IV in electron transport chain was found to be recovered compared with that in HFD group. However, the activity of enzymes of the citric acid cycle, complex II, and citrate synthase did not recover. This suggests that the function of enzymes involved in the citric acid cycle and electron transport chain are differently repaired by the administration of emodin and rhein. Given that the beta-oxidation of fatty acid decomposition is directly linked to the citric acid cycle, it can be speculated that emodin and rhein in C. tora are involved in the amelioration of overall fat metabolism with reduction of circulating triglyceride and fatty acid by fat oxidation. It might result in burdening enzymes in the citric acid cycle before the direct action on the mitochondrial function improvement. In addition, it should be noted that the methodological approach of the current study as assessing the mitochondrial enzyme activity provided a profound analysis of the changes in enzyme function in response to the metabolic state, in comparison to detecting the enzyme expression level by western blotting which exhibits superficial and similar changes in all complexes. In conclusion, administration of the emodin and rhein to HFD mouse successfully enhanced mitochondrial enzymes' activity in the electron transport chain and prevented the compensatory increase of mitochondrial enzyme expression in retina in vivo that should lead to mitochondrial dysfunction and diabetic retinopathy induced by metabolites overload.

\section{Abbreviations}

CSEE: C. tora seed ethanolic extract; ER/CSEE: Emodin and rhein in C. tora seed ethanolic extract; HFD: High-fat diet; T2D: Type 2 diabetes; OXPHOS: Oxidative phosphorylation; NADH: Nicotinamide adenine dinucleotide; BSA: Bovine serum albumin; DCPIP: 2,6-dichlorophenolindolphenol; DTNB: 5,5'-Dithiobis(2-nitrobenzoic acid); TNB: 2-Nitro-5-thiobenzoic acid; PVDF: Polyvinylidene fluoride.

\section{Acknowledgements}

Not applicable.

\section{Authors' contributions}

SS, MC conceived the project and designed the experiments. MU, EK and TH performed the experiments. SS, MC, MU and EK analyzed the data, and EK, and SS wrote the manuscript. All authors read and approved the final manuscript.

\section{Funding}

This work was supported by the National Research Foundation of Korea (Grant Number 2020R1A2C1101110) and by the Korea Institute of Planning and Evaluation for Technology in Food, Agriculture, Forestry (IPET) through High Value-added Food Technology Development Program, funded by the Ministry of Agriculture, Food and Rural Affairs (MAFRA) (Grant Number 313028-3). 


\section{Availability of data and materials}

The datasets used and/or analysed during the current study are available from the corresponding author on reasonable request.

\section{Declarations}

\section{Competing interests}

The authors declare that they have no competing interests.

\section{Author details}

'Department of Biotechnology and Bioengineering, College of Engineering, Chonnam National University, 77 Yongbong-ro, Buk-gu, Gwangju 61186, Republic of Korea. ${ }^{2}$ Research Division of Food Functionality, Korea Food Research Institute, Wanju 55365, Republic of Korea. ${ }^{3}$ Interdisciplinary Program of Bioenergy and Biomaterials Graduate School, College of Engineering, Chonnam National University, Gwangju 61186, Republic of Korea. ${ }^{4}$ Department of Optometry, College of Energy and Biotechnology, Seoul National University of Science and Technology, Seoul 01811, Republic of Korea. ${ }^{5}$ Convergence Institute of Biomaterials and Bioengineering, Seoul National University of Science and Technology, Seoul 01811, Republic of Korea.

Received: 24 February 2021 Accepted: 17 April 2021

Published online: 28 April 2021

\section{References}

1. Wang C-Y, Neil DL, Home P (2018) 2020 vision-an overview of prospects for diabetes management and prevention in the next decade. Diabetes Res Clin Pr 143:101-112

2. Stolar M (2010) Glycemic control and complications in type 2 diabetes mellitus. Am J Med 123(3):S3-S11

3. Hatefi Y (1985) The mitochondrial electron transport and oxidative phosphorylation system. Annu Rev Biochem 54(1):1015-1069

4. Rabøl R, Højberg PM, Almdal T, Boushel R, Haugaard SB, Madsbad S, Dela $F$ (2009) Effect of hyperglycemia on mitochondrial respiration in type 2 diabetes. J Clin Endocr Metab 94(4):1372-1378

5. Martin A, Komada MR, Sane DC (2003) Abnormal angiogenesis in diabetes mellitus. Med Res Rev 23(2):117-145

6. Pugliese G, Tilton RG, Williamson JR (1991) Glucose-induced metabolic imbalances in the pathogenesis of diabetic vascular disease. Diabetes Metab Rev 7(1):35-59

7. Fong DS, Aiello L, Gardner TW, King GL, Blankenship G, Cavallerano JD, Ferris FL, Klein R (2004) Retinopathy in diabetes. Diabetes Care 27(suppl 1):s84-s87

8. Yau JW, Rogers SL, Kawasaki R, Lamoureux EL, Kowalski JW, Bek T, Chen S-J, Dekker JM, Fletcher A, Grauslund J (2012) Global prevalence and major risk factors of diabetic retinopathy. Diabetes Care 35(3):556-564

9. Jain S, Patil U (2010) Phytochemical and pharmacological profile of Cassia tora Linn.-An overview. Indian J of Nat Prod Resour 1:430-437

10. Hatano T, Uebayashi H, Ito H, Shiota S, Tsuchiya T, Yoshida T (1999) Phenolic constituents of Cassia seeds and antibacterial effect of some naphthalenes and anthraquinones on methicillin-resistant Staphylococcus aureus. Chem Pharm Bull 47(8):1121-1127

11. El-Halawany AM, Chung MH, Nakamura N, Ma C-M, Nishihara T, Hattori $M$ (2007) Estrogenic and anti-estrogenic activities of Cassia tora phenolic constituents. Chem Pharm Bull 55(10):1476-1482

12. Nam J, Choi H (2008) Effect of butanol fraction from Cassia tora L. seeds on glycemic control and insulin secretion in diabetic rats. Nutr Res Pract 2(4):240-246

13. Patil UK, Saraf S, Dixit V (2004) Hypolipidemic activity of seeds of Cassia tora Linn. J Ethnopharmacol 90(2-3):249-252

14. Spinazzi M, Casarin A, Pertegato V, Salviati L, Angelini C (2012) Assessment of mitochondrial respiratory chain enzymatic activities on tissues and cultured cells. Nat Protoc 7(6):1235

15. Chang C-H, Lin C-C, Yang J-J, Namba T, Hattori M (1996) Anti-inflammatory effects of emodin from ventilago leiocarpa. Am J Chin Med 24(02):139-142

16. Li H-L, Chen H-L, Li H, Zhang K-L, Chen X-Y, Wang X-W, Kong Q-Y, Liu J (2005) Regulatory effects of emodin on NF-kB activation and inflammatory cytokine expression in RAW 264.7 macrophages. Int J Mol Med 16(1):41-47

17. Zhong X-F, Huang G-D, Luo T, Deng Z-Y, Hu J-N (2012) Protective effect of rhein against oxidative stress-related endothelial cell injury. Mol Med Rep 5(5):1261-1266

18. Aviello G, Rowland I, Gill Cl, Acquaviva AM, Capasso F, McCann M, Capasso R, Izzo AA, Borrelli F (2010) Anti-proliferative effect of rhein, an anthraquinone isolated from Cassia species, on Caco-2 human adenocarcinoma cells. J Cell Mol Med 14(7):2006-2014

19. Araujo TG, de Oliveira AG, Saad MJA (2013) Insulin-resistance-associated compensatory mechanisms of pancreatic beta cells: a current opinion. Front Endocrinol 4:146

20. Montanya $E$ (2014) Insulin resistance compensation: not just a matter of $\beta$-Cells? Diabetes 63(3):832-834

21. Ko E, Um MY, Choi M, Han T, Kim IH, Shin S (2020) Cassia tora seed improves pancreatic mitochondrial function leading to recovery of glucose metabolism. Am J Chin Med 48(03):615-629

22. Han WH, Gotzmann J, Kuny S, Huang H, Chan CB, Lemieux H, Sauve Y (2017) Modifications in retinal mitochondrial respiration precede type 2 diabetes and protracted microvascular retinopathy. Invest Ophth Vis Sci 58(10):3826-3839

23. Kowluru RA, Abbas SN (2003) Diabetes-induced mitochondrial dysfunction in the retina. Invest Ophth Vis Sci 44(12):5327-5334

24. Kowluru RA, Mishra M (2015) Oxidative stress, mitochondrial damage and diabetic retinopathy. BBA-Mol Basis Dis 1852(11):2474-2483

25. Kowluru RA, Atasi L, Ho Y-S (2006) Role of mitochondrial superoxide dismutase in the development of diabetic retinopathy. Invest Ophth Vis Sci 4:1594-1599

\section{Publisher's Note}

Springer Nature remains neutral with regard to jurisdictional claims in published maps and institutional affiliations.

\section{Submit your manuscript to a SpringerOpen ${ }^{\circ}$ journal and benefit from:}

- Convenient online submission

Rigorous peer review

- Open access: articles freely available online

- High visibility within the field

Retaining the copyright to your article

Submit your next manuscript at springeropen.com 